\title{
A 12 week activity point system achieves weight loss in sedentary overweight and obese women: a pilot study
}

\author{
S. Findlay, H. I. M. Davidson, S. Smith and J. Jones \\ Dietetics Nutrition and Biological Sciences, Queen Margaret University, Edinburgh EH21 6UU
}

The recent Scottish Health Survey reported that $65.1 \%$ of adults in Scotland were overweight or obese ${ }^{(1)}$. It is well established that the low levels of activity are associated with increased body weight ${ }^{(2)}$ and the current guidelines for activity are not being met with only $33 \%$ of woman meeting the target of 30 minutes of activity most days of the week ${ }^{(1)}$. The aim of this study was to increase activity levels using a simple, self-guided activity point system ${ }^{(3)}$ and assess changes in body weight.

Overweight and obese women $\left(\mathrm{BMI}>25 \mathrm{~kg} / \mathrm{m}^{2}\right)$ were recruited from the University. to participate in a 12 week intervention programme. Participants were instructed to increase activity using the point system and this was recorded using a daily self-reported activity diary. Participants were reviewed at baseline, 6 weeks and 12 weeks when body weight, body mass index and actual activity levels were measured using an activPAL accelerometer. Activity was categorised as sitting/lying, standing or stepping. Paired t-tests were used to compare the changes from baseline to 12 weeks.

Seven women mean age 46 SD 6.7 years completed the intervention programme. Following completion significant reductions in both BMI (mean $34.7 \mathrm{SD} 8.7$ v. $34.0 \mathrm{SD} 8.8 \mathrm{~kg} / \mathrm{m}^{2}$ ) and body weight (mean $93.6 \mathrm{SD} 28.5$ v. $91.7 \mathrm{sD} 28.6 \mathrm{~kg}$ ) were evident $p<0.01$. Results in table show changes in time spent stepping, standing and sitting/lying from baseline to 12 weeks.

\begin{tabular}{lccccc}
\hline & \multicolumn{2}{c}{ Baseline } & & \multicolumn{2}{c}{12 weeks } \\
\cline { 2 - 3 } \cline { 5 - 6 } & mean & SD & & mean & SD \\
\hline Sitting/lying (hours) & 19.83 & 1.18 & & $19.02^{*}$ & 1.66 \\
Standing (hours) & 2.85 & 0.73 & & $3.55^{*}$ & 1.19 \\
Stepping (hours) & 1.31 & 0.46 & & 1.38 & 0.48 \\
\hline * Significantly different from baseline $p<0.05$ & & &
\end{tabular}

These results indicate that following a simple activity point system is an effective method to reduce inactivity in an overweight and obese female population. Whilst the activity point system did result in lower levels of sitting/lying times it was not apparent that this translated into increased levels of activities such as stepping as the resultant effect was an increase in standing time only. However this model for reducing inactivity shows potential as a method of weight reduction, as despite being a pilot study, significant reductions in both body weight and BMI were evident. It remains to be elucidated whether these results can be generalised to the population as a whole and whether improvements in diet quality contribute to the weight loss seen.

1. Scottish Government (2010) http://www.scotland.gov.uk/Resource/Doc/358842/0121284.pdf

2. Physical Activity Guidelines Advisory Committee Report 2008. www.health.gov/PAGuidelines/committeereport.aspx

3. Ehrsam R, Hoerler-Koerner U, Stoffel S et al. (2009): British Journal of Sports Medicine 43; $951-953$. 